\title{
Reanalysis-based optimal design of trusses
}

\author{
Liang-Jenq Leu ${ }^{*, \dagger, \ddagger}$ and Chang-Wei Huang ${ }^{\S}$ \\ Department of Civil Engineering, National Taiwan University, Taipei 10617, Taiwan, R.O.C.
}

\begin{abstract}
SUMMARY
This paper presents a structural reanalysis method and its applications in optimal design of trusses. This reanalysis technique is derived primarily on the basis of a reduced basis formulation, and it has several advantages over previous reduced basis methods. In particular, the reduced system is uncoupled by using a Gram-Schmidt orthonormalization procedure and an error measure is introduced to adaptively monitor whether a good approximate solution is achieved. The latter aspect makes this reanalysis method suitable for use in optimal design problems because the changes in design variables usually vary during a design process. Discussions are presented on the implementation of this reanalysis method using both mathematical programming and optimality criteria-based optimization schemes. Finally, several example problems of optimal truss design are used to validate the proposed reanalysis-based design procedure. The presented numerical results indicate that the new reanalysis technique affects very slightly the accuracy of the optimal solutions and it does speed up the design process when the system analysed is large. Copyright (C) 2000 John Wiley \& Sons, Ltd.
\end{abstract}

KEY WORDS: reanalysis; reduced-basis formulation; optimal design; mathematical programming; optimality criteria; truss

\section{INTRODUCTION}

The subject of design optimization has attracted substantial attention recently [1-3]. During an optimization process, the optimal solution is achieved iteratively, with several repeated analyses for intermediate designs are required. The analysis is computation expensive if the system analysed is of large scale. Therefore, an accurate and efficient reanalysis method is very desirable to speed up the design process without affecting the accuracy of the optimal solution.

Previous studies on reanalysis using the finite element method (FEM) or the boundary element method (BEM) have mostly focused only on reanalysis problems and little attention has been paid to the issue of whether a reanalysis method is able to enhance the efficiency of the design

\footnotetext{
${ }^{*}$ Correspondence to: Liang-Jenq Leu, Department of Civil Engineering, National Taiwan University, Taipei, Taiwan 10617, Republic of China

†E-mail: 1jleu@ce.ntu.edu.tw

¥Associate Professor

$\S$ Graduate Research Student
}

Contract/grant sponsor: National Science Council of the Republic of China; contract/grant numbers: NSC-85-2211-E-002013, NSC-86-2221-E-002-076 
process. Moreover, even for reanalysis problems, the issue of accuracy is more emphasized than the issue of efficiency in those studies. For example, in the FEM context, Taye [4], and Kirsch $[5,6]$ proposed accurate reduced basis methods for structural reanalysis without addressing the question of efficiency. In the BEM literature, Kane et al. [7] and Saigal [8] developed reanalysis techniques using iterative methods; efficiency was discussed only for reanalysis problems and not addressed for optimization application. Very recently, Leu [9] proposed a reduced basis formulation for boundary element systems, providing significant increase of speed in the reanalysis application of this method. Since the most important reason for using a reanalysis method is to enhance the efficiency of an optimal design process, hence the efficiency and accuracy of a reanalysis method should be evaluated using optimal design problems. To this end, Leu [10] applied the reanalysis technique for boundary element systems developed previously by Leu [9] to carry out shape optimization problems. Efficiency was enhanced for such problems, although the gain in efficiency for optimization problems was not as significant compared with the gain for reanalysis problems.

The objective of this paper is twofold. First, a reduced basis method extended from Kirsch's method is presented. This improved version is suitable for use in design optimization problems since the required number of basis vectors for each reanalysis can be automatically determined with less cost. The second goal is to investigate how efficient the proposed reanalysis technique is when applied to optimal design of trusses. For this purpose, the procedures of implementing the proposed reanalysis method in both mathematical programming (MP) and optimality criteria(OC)based optimizers are then discussed. Finally, several example problems are used to validate the proposed reanalysis-based design procedure.

\section{REDUCED BASIS METHOD FOR REANALYSIS}

\subsection{Reduced system}

Define a reference system as the system whose solution has been obtained exactly using a direct method such as Gaussian elimination or the triple factoring method [11]. This is the system to which later analyses using the reduced basis technique are referred. At the beginning of a design process, the reference system is taken to be the one corresponding to the original design. Later it may be updated, as discussed in Section 2.4.

Let the reference system be expressed as

$$
\mathbf{K}_{0} \mathbf{U}_{0}=\mathbf{P}_{0}
$$

where $\mathbf{K}_{0}, \mathbf{U}_{0}$ and $\mathbf{P}_{0}$ are the stiffness matrix, displacement and load vectors, respectively. Assuming that the triple factoring method is employed to solve Equation (1), the system matrix, therefore, can be factored as

$$
\mathbf{K}_{0}=\mathbf{L}_{0} \mathbf{D}_{0} \mathbf{L}_{0}^{\mathrm{T}}
$$

where $\mathbf{L}_{0}$ is a lower triangular matrix and $\mathbf{D}_{0}$ is a diagonal matrix. The stiffness matrix is stored using the skyline format (variable band width).

Since in design optimization, an analysis is performed for each intermediate (modified) design, let the corresponding system be written as

$$
\mathbf{K} \mathbf{U}=\left(\mathbf{K}_{0}+\Delta \mathbf{K}\right)\left(\mathbf{U}_{0}+\Delta \mathbf{U}\right)=\left(\mathbf{P}_{0}+\Delta \mathbf{P}\right)=\mathbf{P}
$$

Copyright (C) 2000 John Wiley \& Sons, Ltd.

Int. J. Numer. Meth. Engng 2000; 49:1007-1028 
Equation (3) can, of course, be solved by a direct method, but that is inefficient when the halfbandwidth of the system is large. An approximate but efficient method, based on a reduced basis formulation, is presented herein to solve Equation (3). Note that the load vector for the modified system, $\mathbf{P}$, can be different from the load vector for the original design, $\mathbf{P}_{0}$.

The main idea of using a reduced basis method to solve Equation (3) is to reduce the number of degrees of freedom by an approximation for the unknowns as follows:

$$
\mathbf{U}_{n \times 1} \approx \tilde{\mathbf{U}}_{n \times 1}=\sum_{i=1}^{m} c_{i} \boldsymbol{\phi}_{i}=\boldsymbol{\Phi}_{n \times m} \mathbf{c}_{m \times 1}
$$

where $\tilde{\mathbf{U}}$ denotes an approximation of $\mathbf{U}, \boldsymbol{\Phi}$ is a matrix composed of $m$ basis vectors, and $\mathbf{c}$ is a column vector composed of the generalized coefficients $c_{i}(i=1, \ldots, m)$. The generation of basis vectors, $\phi_{i}$, will be discussed later.

The reduced system corresponding to Equation (3) can be derived as follows. Substitute Equation (4) into Equation (3) and then premultiply both sides by $\boldsymbol{\Phi}^{\mathrm{T}}$ to obtain

$$
\boldsymbol{\Phi}^{\mathrm{T}} \mathbf{K} \boldsymbol{\Phi} \mathbf{c}=\boldsymbol{\Phi}^{\mathrm{T}} \mathbf{P}
$$

Note that Equation (5) is a system of order $m$, which is usually much smaller than that of the original system, i.e. $n$. The reduced system can be solved by a direct method such as the triple factoring method. After solving for $\mathbf{c}$, the approximate solution can then be obtained using Equation (4).

However, when using Equation (4) the question arises: How many basis vectors should be used in approximating $\mathbf{U}$ to achieve a prescribed level of accuracy? One direct approach is to increase the number of basis vectors by one each time until a defined error measure is smaller than a preset tolerance. For example, an error measure $E_{p}$ may be defined as

$$
E_{p}=\frac{\|\mathbf{K} \tilde{\mathbf{U}}-\mathbf{P}\|}{\|\mathbf{P}\|}
$$

where $\|\cdot\|$ denotes the $L_{2}$ norm. However, it takes a fairly large computational effort to evaluate Equation (6). Also, the evaluation needs to be repeated each time when the number of basis vectors is increased. Therefore, the use of Equation (5) together with Equation (6) is inefficient. In addition, as the number of basis vectors is increased the reduced system may become illconditioned, making it difficult to obtain an accurate solution in this case.

\subsection{Uncoupled reduced system and convergence criterion}

In the following, a Gram-Schmidt orthonormalization procedure is employed to generate a new set of basis vectors $\psi_{i}$ from $\phi_{i}$ such that

$$
\boldsymbol{\psi}_{i}^{\mathrm{T}} \mathbf{K} \boldsymbol{\psi}_{j}=\delta_{i j} \quad \text { or } \quad \boldsymbol{\Psi}^{\mathrm{T}} \mathbf{K} \boldsymbol{\Psi}=\mathbf{I}
$$

where $\delta_{i j}$ is the Kronecker delta and $\mathbf{I}$ is the identity matrix. This procedure is detailed in Table I.

If $\boldsymbol{\Psi}$ are adopted as the basis vectors, the associated reduced system of Equation (5) becomes

$$
\mathbf{c}=\boldsymbol{\Psi}^{\mathrm{T}} \mathbf{P} \quad \text { or } \quad c_{i}=\boldsymbol{\psi}_{i}^{\mathrm{T}} \mathbf{P}
$$


Table I. Generation of orthonormal basis vectors with respect to $\mathbf{K}$.

\begin{tabular}{lc}
\hline (a) If $i=1$, & $\boldsymbol{\psi}_{1}=\boldsymbol{\phi}_{1} /\left(\boldsymbol{\phi}_{1}^{\mathrm{T}} \mathbf{K} \boldsymbol{\phi}_{1}\right)^{1 / 2}$ \\
(b) If $i>1$, & $\overline{\boldsymbol{\psi}}_{i}=\boldsymbol{\phi}_{i}-\sum_{j=1}^{i-1}\left(\boldsymbol{\phi}_{i}^{\mathrm{T}} \mathbf{K} \boldsymbol{\psi}_{j}\right) \boldsymbol{\psi}_{j}$ \\
& $\boldsymbol{\psi}_{i}=\overline{\boldsymbol{\psi}}_{i} /\left(\overline{\boldsymbol{\psi}}_{i}^{\mathrm{T}} \mathbf{K} \overline{\boldsymbol{\psi}}_{i}\right)^{1 / 2}$
\end{tabular}

Thus, the reduced system becomes uncoupled. Then, from Equation (4), $\mathbf{U}$ can be approximated as

$$
\tilde{\mathbf{U}}=\sum_{i=1}^{m} c_{i} \boldsymbol{\psi}_{i}=\sum_{i=1}^{m}\left(\boldsymbol{\psi}_{i}^{\mathrm{T}} \mathbf{P}\right) \boldsymbol{\psi}_{i}
$$

On the basis of the above uncoupled system, a computation-inexpensive criterion for adaptively determining the required number of basis vectors is proposed herein. We begin with the $m$-term approximation $\tilde{\mathbf{U}}=\sum_{i=1}^{m} c_{i} \boldsymbol{\psi}_{i}$. As one would expect, the contribution of the last term, $c_{m} \boldsymbol{\psi}_{m}$, to $\tilde{\mathbf{U}}$ becomes very small when $\tilde{\mathbf{U}}$ converges. Therefore, the required number of basis vectors $m$ may be chosen such that

$$
E_{u}=\frac{\left\|c_{m} \psi_{m}\right\|}{\sum_{i=1}^{m}\left\|c_{i} \psi_{i}\right\|}<e_{u}
$$

where $e_{u}$ is a given tolerance. This criterion can be approximated by

$$
E_{\mathrm{c}}=\frac{\left|c_{m}\right|}{\sum_{i=1}^{m}\left|c_{i}\right|}<e_{\mathrm{c}}
$$

where $e_{\mathrm{c}}$ is a given tolerance associated with $E_{\mathrm{c}}$. The approximation is proper because Equation (7) implies that the magnitudes of $\left\|\psi_{i}\right\|$ are of about the same order.

\subsection{Generation of the original basis vectors}

As for the selection of the original basis vectors $\phi_{i}$, Kirsch's method [5] is followed. First, premultiply Equation (3) by $\mathbf{K}_{0}^{-1}$ to produce

$$
(\mathbf{I}+\mathbf{B}) \mathbf{U}=\mathbf{U}_{p}
$$

where

$$
\begin{aligned}
\mathbf{B} & =\mathbf{K}_{0}^{-1} \Delta \mathbf{K} \\
\mathbf{U}_{p} & =\mathbf{K}_{0}^{-1} \mathbf{P}
\end{aligned}
$$

In the above, $\mathbf{K}_{0}^{-1}$ is the inverse of $\mathbf{K}_{0}$, and $\Delta \mathbf{K}=\mathbf{K}-\mathbf{K}_{0}$. Note that $\mathbf{K}_{0}^{-1}$ works as a preconditioning matrix in solving Equation (3).

It can be derived from Equation (12) that

$$
\mathbf{U}=(\mathbf{I}+\mathbf{B})^{-1} \mathbf{U}_{p}=\left(\mathbf{I}-\mathbf{B}+\mathbf{B}^{2}-\mathbf{B}^{3}+\cdots\right) \mathbf{U}_{p}
$$


Table II. Proposed reanalysis procedure.

1. Initialization: Calculate $\mathbf{U}_{p}\left(=\mathbf{K}_{0}^{-1} \mathbf{P}\right)$; let $i=1$ and the initial solution $\tilde{\mathbf{U}}_{0}=\mathbf{0}$.

2. Generate $\phi_{i}$ using Equation (16) and orthonormalize $\phi_{i}$ according to Table I to obtain $\psi_{i}$.

3. Calculate $c_{i}$ using Equation (8).

4. Update solution: $\tilde{\mathbf{U}}_{i}=\tilde{\mathbf{U}}_{i-1}+c_{i} \boldsymbol{\psi}_{i}$.

5. Check convergence:

Compute $E_{p}$ using Equation (6) if $i=1$ and $E_{\mathrm{c}}$ using Equation (11) if $i>1$; check whether they are less than the specified tolerances $e_{p}$ and $e_{\mathrm{c}}$, respectively. If yes, stop; otherwise let $i=i+1$ and go to step 2 .

This series is called Neumann series. It converges if and only if the spectral radius of $\mathbf{B}$ is smaller than one [12]. Even if it does converge, the convergence rate may be slow. Therefore, taking a few terms of Equation (15) to approximate $\mathbf{U}$ is not efficient. Instead, Kirsch adopted the terms in the series as the basis vectors. Namely, the choice of $\boldsymbol{\Phi}$ is

$$
\boldsymbol{\Phi}=\left[\boldsymbol{\phi}_{1}, \boldsymbol{\phi}_{2}, \boldsymbol{\phi}_{3}, \ldots\right]=\left[\mathbf{U}_{p}, \mathbf{B} \mathbf{U}_{p}, \mathbf{B}^{2} \mathbf{U}_{p}, \ldots\right]
$$

Notice that the generation of $\phi_{i}$ according to Equation (16) is computation-inexpensive. This is because

$$
\boldsymbol{\phi}_{i}=\mathbf{B} \boldsymbol{\phi}_{i-1} \quad(i>1)
$$

With the definition of $\mathbf{B}$ given in Equation (13), this becomes

$$
\phi_{i}=\mathbf{K}^{-1} \Delta \mathbf{K} \phi_{i-1} \quad \text { or } \quad \mathbf{K}_{0} \boldsymbol{\phi}_{i}=\Delta \mathbf{K} \boldsymbol{\phi}_{i-1} \quad(i>1)
$$

Since the factored form of $\mathbf{K}_{0}$ has been given in Equation (2), the calculation of $\phi_{i}(i>1)$ using Equation (18) is computation inexpensive since it involves only the multiplication of $\Delta \mathbf{K}$ and $\phi_{i-1}$, followed by a backward and a forward substitution.

\subsection{Summary of the proposed reanalysis procedure}

From the above discussions, the reanalysis procedure using the proposed reduction method can be summarized in Table II.

Note that in the above procedure $E_{p}$ is still needed to check convergence when $i=1$ since in this case the use of $E_{\mathrm{c}}$ as a convergence indicator is unsuitable. The values $e_{p}=0.001$ and $e_{\mathrm{c}}=0.01$ used in the numerical examples presented later are suggested according to several numerical experiments by Huang [13].

Note that in this study the maximum number of basis vectors allowed is 10 . If convergence has not been achieved when 10 basis vectors are used, the system currently being solved using the reanalysis method needs to be solved again using the triple factoring method. The system is then taken as the new reference system, i.e. the system described by Equation (1). It is very possible that in this case the changes in the design variables between the current design and the reference design are relatively large. Hence, if the reference system is updated, latter reanalyses may require fewer basis vectors. The maximum number of 10 is a good choice from our experience.

Several differences between the proposed reanalysis method and Kirsch's method are pointed out here. First, Kirsch's method considers only the case where $\mathbf{P}=\mathbf{P}_{0}$; namely, the original design and the modified design have the same load vector. However, this study has extended the method 
to the case where $\mathbf{P} \neq \mathbf{P}_{0}$. This extension is needed to calculate displacement sensitivities using the reanalysis technique, as will be discussed in Section 3.3. In addition, Kirsch's method does not employ the Gram-Schmidt orthonormalization procedure to uncouple the reduced system as described in Section 2.2; its associated reduced system simply takes the form of Equation (5). Finally, the adaptive computation-inexpensive convergence criterion as described by Equation (11) is also new.

It should be mentioned that the proposed reanalysis technique can also deal with the case where the modified design results from eliminating part of the members of the original design; this occurs in the problem of optimal topology design. Actually, Kirsch's method has been applied to this type of problem [14]. The proposed reanalysis method gives results, although not reported here, as accurate as those reported in Reference [14].

\section{REANALYSIS-BASED OPTIMAL DESIGN USING MP METHODS}

\subsection{Design formulation}

For truss structures, a design optimization problem usually aims at seeking a least-weight structure while satisfying all the stress and displacement constraints. Mathematically, it may be stated as

$$
\text { minimize } \quad f(\mathbf{d})=\sum_{i=1}^{N_{m}} A_{i} L_{i} \rho_{i}
$$

subject to

$$
\begin{array}{ll}
\sigma_{i}^{\ell} \leqslant \sigma_{i} \leqslant \sigma_{i}^{\mathrm{u}} & \left(i=1, \ldots, N_{s}\right) \\
u_{i}^{\ell} \leqslant u_{i} \leqslant u_{i}^{\mathrm{u}} & \left(i=1, \ldots, N_{u}\right) \\
d_{i}^{\ell} \leqslant d_{i} \leqslant d_{i}^{\mathrm{u}} & \left(i=1, \ldots, N_{d}\right)
\end{array}
$$

Equation (19) defines the weight of the structure, $N_{m}$ being the number of members, and $A_{i}, L_{i}$, and $\rho_{i}$ are, respectively, the cross-sectional area, length, and density of the $i$ th member. Equations (20)-(22) denote, respectively, the stress, displacement, and side constraints, where a superscript ' $u$ ' indicates upper bounds and a superscript ' $\ell$ ' indicates lower bounds. Also, $N_{s}, N_{u}, N_{d}$, are the numbers of the stress, displacement and side constraints, respectively. The design variable vector d may contain, for example, the cross-sectional areas of members for sizing design problems, the co-ordinates of nodes for configuration design problems. Generally speaking, the objective function $f$ and the stress and displacement constrains are implicit functions of $\mathbf{d}$.

Two types of methods are commonly used in solving the above optimization problems. One type is called MP methods, being based on mathematical programming; the other type is called OC methods, being based on optimality criteria, i.e. Kuhn-Tucker conditions. These two classes of methods will be employed in conjunction with the proposed reanalysis method to carry out optimal design of trusses. The MP method is discussed in this section, while the OC method is detailed in next section.

\subsection{MP Optimizer}

In general, an optimal solution to the problem stated in Section 3.1 is achieved in an iterative manner through the use of an optimizer. Typically, a MP optimizer uses linear or non-linear 
programming to propose a new design by providing a better value of the objective function without violating the constraints of the problem. In each design cycle, say the $k$ th step, the input to the optimizer includes $d_{i}^{(k)}, d_{i}^{\ell}, d_{i}^{\mathrm{u}}\left(i=1, \ldots, N_{d}\right), f\left(\mathbf{d}^{(k)}\right), \sigma_{i}^{(k)}, \sigma_{i}^{\ell}, \sigma_{i}^{\mathrm{u}}\left(i=1, \ldots, N_{s}\right), u_{i}^{(k)}$, $u_{i}^{\ell}, u_{i}^{\mathrm{u}}\left(i=1, \ldots, N_{u}\right)$, and possibly the gradients of the objective function and constraints with respect to $\mathbf{d}: \partial f / \partial d_{i}\left(i=1, \ldots, N_{d}\right), \partial \sigma_{i} / \partial d_{j}\left(i=1, \ldots, N_{s}, j=1, \ldots, N_{d}\right)$, and $\partial u_{i} / \partial d_{j}(i=$ $\left.1, \ldots, N_{u}, j=1, \ldots, N_{d}\right)$, also evaluated at $\mathbf{d}^{(k)}$. The optimizer then generates a new set of design variables $\mathbf{d}^{(k+1)}$ and indicates whether the gradients of the objective function and constraints are needed in the next design cycle. If the new design is acceptable, the process stops. Otherwise, the iterative process is continued, producing a succession of designs, until an optimal design is obtained.

There are a variety of reliable MP methods and also many computer programs have been developed based on these methods. The MP optimizer adopted here, subroutine DN0ONF, is available from the IMSL/MATH library [15]. This subroutine is based on the subroutine NLPQL, a FORTRAN code developed by Schittkowski [16]. A brief description of the algorithm is given below. Further details of the algorithm can be found in the above references.

For the optimization problems formulated in Section 3.1, the sequential quadratic programming algorithm DN0ONF uses a quadratic approximation of the Lagrangian and linearization of the constraints to define a sequence of subproblems. This requires the evaluation of a positive definite approximation of the Hessian of $f$. Let $\mathbf{v}^{(k)}$ be the solution of a subproblem at the $k$ th iterative step. A line search is used to find a new design $\mathbf{d}^{(k+1)}$, defined as

$$
\mathbf{d}^{(k+1)}=\mathbf{d}^{(k)}+\lambda^{(k)} \mathbf{v}^{(k)}, \quad 0<\lambda^{(k)}<1 \quad(\text { no sum on } k)
$$

such that the augmented Lagrangian function has a lower function value at the new design. Here, $\lambda^{(k)}$ is the step length parameter. The iterative process stops when the Kuhn-Tucker optimality conditions are satisfied within an acceptable tolerance.

\subsection{Sensitivity calculation using the proposed reduced basis technique}

Differentiate Equation (3) with respect to each design variable and rearrange the terms to yield

$$
\mathbf{K} \frac{\partial \mathbf{U}}{\partial d_{j}}=-\frac{\partial \mathbf{K}}{\partial d_{j}} \mathbf{U} \quad\left(j=1, \ldots, N_{d}\right)
$$

where the condition $\partial \mathbf{P} / \partial d_{j}=\mathbf{0}$ has been assumed, which is usually the case in structural design. Namely, the applied loads are independent of the design variables and therefore $\mathbf{P}$ of Equation (3) remains unchanged during the design process and is equal to $\mathbf{P}_{0}$ of Equation (1). In Equation (23), $\partial \mathbf{K} / \partial d_{j}$ can be obtained by assembling the gradients of each member's stiffness matrix. The stiffness matrix for a spatial truss element is given in the appendix for completeness; its gradients with respect to either cross-sectional areas or co-ordinates of the nodes can be easily derived as the stiffness matrix is given in an explicit form [13].

Conventionally, a direct method such as the triple factoring method is utilized to solve for $\mathbf{U}$ from Equation (3), then substituting $\mathbf{U}$ into Equation (23) to form the right-hand side. Finally, the displacement gradients $\partial \mathbf{U} / \partial d_{j}$ can be obtained by a backward substitution followed by a forward one. However, if the proposed reduced basis method is adopted to solve Equation (3), then the above strategy is not applicable since the factored form of $\mathbf{K}$ is not available. Comparing Equation (23) with Equation (3), one can find that the reduced basis method can also be employed to solve for $\partial \mathbf{U} / \partial d_{j}$. This is because if one lets $\mathbf{P}$ of Equation (3) be equal to $-\left(\partial \mathbf{K} / \partial d_{j}\right) \mathbf{U}$ and replaces 
the unknowns $\mathbf{U}$ by $\partial \mathbf{U} / \partial d_{j}$, then Equation (3) becomes Equation (23). Namely, Equation (23) can also be solved with respect to the reference system given in Equation (1) using the proposed reanalysis method.

Denote the axial stress and its gradients for a generic member, say the $i$ th member, as $\sigma_{i}$ and $\partial \sigma_{i} / \partial d_{j}\left(j=1, \ldots, N_{d}\right)$, respectively. Then, $\sigma_{i}$ can be obtained from the nodal displacements $\mathbf{U}_{i}$ of the same member using the below relation

$$
\sigma_{i}=\mathbf{S}_{i} \mathbf{U}_{i}
$$

Notice that $\mathbf{U}_{i}$ is a subset of $\mathbf{U}$ and for a spatial truss element

$$
\mathbf{S}_{i}=\frac{E_{i}}{L_{i}^{2}}\left\langle-D_{x i},-D_{y i},-D_{z i}, D_{x i}, D_{y i}, D_{z i}\right\rangle
$$

where $E_{i}$ is Young's modulus, $L_{i}$ the element's length, $D_{x i}=X_{2 i}-X_{1 i}, D_{y i}=Y_{2 i}-Y_{1 i}, D_{z i}=Z_{2 i}-Z_{1 i}$; $\left(X_{1 i}, Y_{1 i}, Z_{1 i}\right)$ and $\left(X_{2 i}, Y_{2 i}, Z_{2 i}\right)$ are the co-ordinates of the two end nodes of the element.

Differentiate Equation (24) with respect to each design variable to produce

$$
\frac{\partial \sigma_{i}}{\partial d_{j}}=\frac{\partial \mathbf{S}_{i}}{\partial d_{j}} \mathbf{U}_{i}+\mathbf{S}_{i} \frac{\partial \mathbf{U}_{i}}{\partial d_{j}} \quad\left(j=1, \ldots, N_{d}\right)
$$

Stress gradients can be calculated by this equation. The procedures for obtaining $\mathbf{U}_{i}, \mathbf{S}_{i}$ and $\partial \mathbf{U}_{i} / \partial d_{j}$ have been discussed above, where $\partial \mathbf{U}_{i} / \partial d_{j}$ is a subset of $\partial \mathbf{U} / \partial d_{j}$. The remaining term $\partial \mathbf{S}_{i} / \partial d_{j}$ can be derived easily for the design variables of areas or co-ordinates of the nodes as $\mathbf{S}_{i}$ is given explicitly in Equation (25).

According to the above discussions, the differences between the conventional approach and the proposed reanalysis-based one for design optimization have two aspects. First, the conventional approach solves for $\mathbf{U}$ from Equation (3) by the triple factoring method, whereas this task is carried out using the reduced basis technique in the present approach. Second, the conventional approach obtains $\partial \mathbf{U} / \partial d_{j}$ from Equation (23) by a forward substitution followed by a backward one, but the present approach employs the reduced basis technique to solve the same equation. Therefore, for a design iteration step where the gradients of the objective function and constraints are needed, the conventional approach requires one decomposition of $\mathbf{K},\left(N_{d}+1\right)$ forward and $\left(N_{d}+1\right)$ backward substitutions, while the present approach needs to use the reduced basis technique $\left(N_{d}+1\right)$ times. As the forward and backward substitutions are computation-inexpensive, the computational effort in solving Equation (23) using the conventional approach is usually less than that using the reanalysis method unless the system is of large scale. Therefore, as the number of design variables increases, the overall efficiency in design optimization on the basis of the proposed reanalysis method decreases even though the reanalysis method may be much faster than the conventional approach when solving Equation (3).

\section{REANALYSIS-BASED OPTIMAL DESIGN USING OC METHODS}

\subsection{Kuhn-Tucker conditions}

The OC optimizer adopted in this study is an improved version of the algorithm originally developed in References [17, 18]; some modifications are made below to the original formulation to obtain a faster convergence rate. For completeness, necessary derivations are repeated 
below. The main reason to choose this particular algorithm is that there is no need to calculate the gradients of the stress and displacement constraints. Such calculations, as discussed in Section 3.3, will lower the efficiency of a reanalysis-based optimization process and should be avoided. However, this optimizer is limited in that it is applicable only to optimal sizing design of trusses.

Since only the cross-sectional areas are taken as the design variables when this OC optimizer is used, the objective function of Equation (19) can be rewritten as

$$
\operatorname{minimize} f(\mathbf{A})=\sum_{i=1}^{N_{d}} A_{i} \bar{L}_{i} \rho_{i}
$$

where $\bar{L}_{i}$ is the sum of the lengths of the members having the same cross-sectional area $A_{i}$. In addition to the inequality constraints as given in Equations (20)-(22), the equilibrium equations as given in Equation (3) are also treated as equality constraints in this formulation.

The Lagrangian for the stated design problem then takes the form

$$
\begin{aligned}
\mathscr{L}(\mathbf{A}, \mathbf{U}, \boldsymbol{\lambda})= & \sum_{i=1}^{N_{d}} A_{i} \bar{L}_{i} \rho_{i}+\left(\boldsymbol{\lambda}_{e}\right)^{\mathrm{T}}(\mathbf{K} \mathbf{U}-\mathbf{P})+\boldsymbol{\lambda}_{u u}^{\mathrm{T}}\left(\mathbf{U}-\mathbf{U}^{\mathrm{u}}\right) \\
& +\boldsymbol{\lambda}_{u l}^{\mathrm{T}}\left(\mathbf{U}^{\ell}-\mathbf{U}\right)+\boldsymbol{\lambda}_{s u}^{\mathrm{T}}\left(\mathbf{S U}-\boldsymbol{\sigma}^{\mathrm{u}}\right)+\boldsymbol{\lambda}_{s l}^{\mathrm{T}}\left(\boldsymbol{\sigma}^{\ell}-\mathbf{S U}\right)
\end{aligned}
$$

where $\left(\boldsymbol{\lambda}_{e}\right)_{n \times 1},\left(\boldsymbol{\lambda}_{u u}\right)_{n \times 1},\left(\boldsymbol{\lambda}_{u l}\right)_{n \times 1},\left(\boldsymbol{\lambda}_{s u}\right)_{N_{s} \times 1}$, and $\left(\boldsymbol{\lambda}_{s l}\right)_{N_{s} \times 1}$ are, respectively, the Lagrange multipliers associated with the equality constraints, displacement constraints of the lower- and upperbound types, and stress constraints of the lower- and upper-bound types; $\lambda$ denotes the collection of all the above Lagrange multipliers. Clearly, the side constraints stated by Equation (22) are not included in the Lagrangian, which is quite common for an $\mathrm{OC}$ algorithm. Note that for convenience either the lower-bound type or the upper-bound type displacement constraints are expressed in terms of $\mathbf{U}$. Hence, in $\boldsymbol{\lambda}_{u u}$ and $\boldsymbol{\lambda}_{u l}$ only the components associated with the displacements that have specified bounds as given in Equation (21) may be non-zero, the other components are zero. Also, $(\mathbf{S})_{N_{s} \times n}$ is the matrix that relates nodal displacements to member stresses, and it can be obtained by assembling $\mathbf{S}_{i}$ of Equation (25). Finally, $\boldsymbol{\sigma}^{\mathrm{u}}$ and $\boldsymbol{\sigma}^{\ell}$ are, respectively, the collection of $\sigma_{i}^{\mathrm{u}}$ and $\sigma_{i}^{\ell}\left(i=1,2, \ldots, N_{s}\right)$; similar definitions for $\mathbf{U}^{\mathrm{u}}$ and $\mathbf{U}^{\ell}$.

The Kuhn-Tucker conditions [19] or optimality conditions require that the inequality constraints of Equations (20), (21) and the equality constraints of Equation (3) be satisfied and also that

$$
\begin{array}{cl}
\frac{\partial \mathscr{L}}{\partial A_{i}}=\rho_{i} \bar{L}_{i}+\boldsymbol{\lambda}_{e}^{\mathrm{T}} \frac{\partial \mathbf{K}}{\partial A_{i}} \mathbf{U}=0 \quad\left(i=1, \ldots, N_{d}\right) \\
\frac{\partial \mathscr{L}}{\partial \mathbf{U}}=\mathbf{K} \boldsymbol{\lambda}_{e}+\boldsymbol{\lambda}_{u u}-\boldsymbol{\lambda}_{u l}+\mathbf{S}^{\mathrm{T}} \boldsymbol{\lambda}_{s u}-\mathbf{S}^{\mathrm{T}} \boldsymbol{\lambda}_{s l}=\mathbf{0} \\
\left(\boldsymbol{\lambda}_{u u}\right)_{i}\left(\mathbf{U}-\mathbf{U}^{\mathrm{u}}\right)_{i}=0 \quad(i=1, \ldots, n) \\
\left(\boldsymbol{\lambda}_{u l}\right)_{i}\left(\mathbf{U}^{\ell}-\mathbf{U}\right)_{i}=0 \quad(i=1, \ldots, n) \\
\left(\boldsymbol{\lambda}_{s u}\right)_{i}\left(\mathbf{S} \mathbf{U}-\boldsymbol{\sigma}^{\mathrm{u}}\right)_{i}=0 \quad\left(i=1, \ldots, N_{s}\right) \\
\left(\boldsymbol{\lambda}_{s l}\right)_{i}\left(\boldsymbol{\sigma}^{\ell}-\mathbf{S U}\right)_{i}=0 \quad\left(i=1, \ldots, N_{s}\right)
\end{array}
$$




$$
\begin{aligned}
& \left(\boldsymbol{\lambda}_{u u}\right)_{i} \geqslant 0 ; \quad\left(\boldsymbol{\lambda}_{u l}\right)_{i} \geqslant 0 \\
& \left(\boldsymbol{\lambda}_{s u}\right)_{i} \geqslant 0 ; \quad\left(\boldsymbol{\lambda}_{s l}\right)_{i} \geqslant 0
\end{aligned}
$$

Note that in Equations $(31)-(36),(\cdot)_{i}$ indicates the $i$ th component of a vector.

Since the stiffness matrix of a truss linearly depends on the cross-sectional areas, the following relation holds:

$$
\sum_{i=1}^{N_{d}} \frac{\partial \mathbf{K}}{\partial A_{i}} A_{i}=\mathbf{K}
$$

With the use of Equations (29) and (37), the objective function of Equation (27) can be rewritten as

$$
f(\mathbf{A})=-\left(\mathbf{K} \boldsymbol{\lambda}_{e}\right)^{\mathrm{T}} \mathbf{U}
$$

Further, by making use of Equation (30), this can also be expressed in terms of the Lagrange multipliers associated with the inequality constraints as follows:

$$
f(\mathbf{A})=\lambda_{u u}^{\mathrm{T}} \mathbf{U}-\boldsymbol{\lambda}_{u l}^{\mathrm{T}} \mathbf{U}+\boldsymbol{\lambda}_{s u}^{\mathrm{T}} \mathbf{S} \mathbf{U}-\boldsymbol{\lambda}_{s l}^{\mathrm{T}} \mathbf{S} \mathbf{U}
$$

Finally for an inequality constraint, either its Lagrange multiplier equals zero or the constraint becomes active, as implied in Equations (31)-(34); therefore Equation (39) can be rewritten as

$$
f(\mathbf{A})=\lambda_{u u}^{\mathrm{T}} \mathbf{U}^{\mathrm{u}}-\boldsymbol{\lambda}_{u l}^{\mathrm{T}} \mathbf{U}^{\ell}+\lambda_{s u}^{\mathrm{T}} \boldsymbol{\sigma}^{\mathrm{u}}-\lambda_{s l}^{\mathrm{T}} \boldsymbol{\sigma}^{\ell}
$$

\subsection{Selection of the most active constraint and scaling of design variables}

For a given set of design variables, the stress and/or displacement constraints may be violated. It is assumed that only one of the above constraints is active during the design iteration process. To this end, a scaling factor is defined as follows:

$$
s^{(k)}=\max \left\{\max _{i=1}^{N_{u}}\left[\frac{u_{i}^{(k)}}{u_{i}^{\mathrm{u}}}, \frac{u_{i}^{\ell}}{u_{i}^{(k)}}\right] ; \max _{i=1}^{N s}\left[\frac{\sigma_{i}^{(k)}}{\sigma_{i}^{\mathrm{u}}}, \frac{\sigma_{i}^{\ell}}{\sigma_{i}^{(k)}}\right]\right\}
$$

where and in the following a bracketed superscript indicates the iteration counter. Notice that $\mathbf{u}$ is a subset of $\mathbf{U}$ having specified displacement bounds. The constraint corresponding to $s^{(k)}$ is defined as the most active constraint. As can be seen from Equation (41), the most active constraint is the constraint that is violated most when some constraints are violated, and it is the constraint closest to the constraint surface when all the constraints are satisfied.

Since the coefficients of the stiffness matrix of a truss are linear functions of the cross-sectional areas, then among all the displacement and stress constraints there will be only one active constraint if the given areas are scaled as follows:

$$
\bar{A}_{i}^{(k)}=A_{i}^{(k)} s^{(k)} \quad\left(i=1, \ldots, N_{d}\right)
$$

Note that $\bar{A}_{i}^{(k)}$ represents the value after scaling. The displacements corresponding to $\bar{A}_{i}^{(k)}$ subjected to the same loading vector are easily shown to be

$$
\bar{U}_{i}^{(k)}=U_{i}^{(k)} / s^{(k)} \quad(i=1, \ldots, n)
$$


The side constraints also need to be checked; therefore

$$
\begin{array}{lll}
\text { If } \bar{A}_{i}^{(k)} \leqslant A_{i}^{\ell} & \text { then } \bar{A}_{i}^{(k)}=A_{i}^{\ell} \quad\left(i=1, \ldots, N_{d}\right) \\
\text { If } \bar{A}_{i}^{(k)} \geqslant A_{i}^{\mathrm{u}} & \text { then } \bar{A}_{i}^{(k)}=A_{i}^{\mathrm{u}} \quad\left(i=1, \ldots, N_{d}\right)
\end{array}
$$

\subsection{Update of Lagrange multipliers}

Since only one inequality constraint is active, as previously assumed, therefore among all the Lagrange multipliers associated with the inequality constraints, only the one corresponding to the most active constraint is non-zero. Namely, there is only one non-zero component in $\lambda_{u u}, \lambda_{u l}, \lambda_{s u}$, and $\lambda_{s l}$. This non-zero component can be obtained by Equation (40), noting that the objective function should be evaluated using the scaled cross-sectional areas $\bar{A}_{i}^{(k)}$ and that on the right-hand side of this equation only the term associated with the most active constraint will be non-zero.

Having obtained the inequality Lagrange multipliers, the equality Lagrange multipliers can be determined as follows:

$$
\overline{\mathbf{K}} \lambda_{e}=-\boldsymbol{\lambda}_{u u}+\boldsymbol{\lambda}_{u l}-\mathbf{S}^{\mathrm{T}} \boldsymbol{\lambda}_{s u}+\mathbf{S}^{\mathrm{T}} \boldsymbol{\lambda}_{s l}
$$

This equation is obtained by rearranging Equation (30); notice that $\mathbf{K}$ has been replaced by $\overline{\mathbf{K}}$, the stiffness matrix corresponding to $\bar{A}_{i}^{(k)}$.

\subsection{Update of design variables}

The formula for updating the design variables is often referred to as the recurrence relation, which for a generic iteration, e.g. the $k$ th step, usually takes the form

$$
A_{i}^{(k+1)}=\bar{A}_{i}^{(k)} \Omega_{i}^{(k)} \quad\left(i=1, \ldots, N_{d}\right)
$$

where $\Omega_{i}^{(k)}$ is called the optimality index. Clearly, $\Omega_{i}^{(k)}$ will approach unity when the design iteration converges.

To introduce the optimality index, a residual value $R_{i}$ is defined first for each design variable as follows:

$$
R_{i}=1+\left(\frac{\lambda_{e}^{\mathrm{T}}\left(\partial \mathbf{K} / \partial A_{i}\right) \mathbf{U}}{\rho_{i} \bar{L}_{i}}\right) \quad\left(i=1, \ldots, N_{d}\right)
$$

As Equation (29) needs to be satisfied for the optimal design, therefore the values of $R_{i}$ will converge to zero. The proposed optimality index is of the form

$$
\begin{aligned}
& \Omega_{i}=1 \quad \text { if }\left|R_{i}\right| \leqslant 10^{-3} \\
& \Omega_{i}=1-\beta \frac{\left|R_{i}\right|}{\sqrt{\left(\sum_{i=1}^{N_{d}} R_{i}^{2}\right)}} \text { if }\left|R_{i}\right|>10^{-3}
\end{aligned}
$$

Equation (49) is applicable since $R_{i}$ would be very small when the optimality conditions are nearly reached. Equation (50) without the denominator is the so-called linear form, and the denominator is introduced to keep a constant initial value of $\beta$ for different design problems. In the literature, $\beta$ is often referred to as an accelerating coefficient. 
If a constant value of $\beta$ is adopted in the design iteration process, it can be found that the value of the objective function will oscillate unless $\beta$ is very small. However, in this case it takes a large number of iterations to achieve the optimal design. To speed up convergence and to keep the oscillation small, it is suggested that the value of $\beta$ be reduced when the oscillation occurs. For this purpose, the following strategy is proposed:

$$
\text { If } \quad\left(f^{(k)}-f^{(k-1)}\right)\left(f^{(k-1)}-f^{(k-2)}\right)<0 \quad(k>2) \quad \text { then } \beta^{(k+1)}=\gamma \beta^{(k)}
$$

where a bracketed superscript indicates the iteration counter, and $\gamma$ is a reduction factor. Khan [20] also used a similar strategy to reduce the oscillation of the objective function. A parametric study was carried out by Huang [13] in order to determine a better combination of the initial value of $\beta$ and $\gamma$, indicating that the use of $\beta=1.8$ and $\gamma=0.7$ is an optimal combination. Note that the above algorithm for updating the design variables is different from those reported in References $[17,18]$ in two aspects. First, in these references, no reduction factor is used and second, different forms of $\Omega_{i}$ are adopted. According to our numerical experiments, the number of iteration to achieve convergence for the proposed algorithm is about one-half of the number required for the algorithms of References [17, 18].

\subsection{Convergence criterion}

The relative change in the objective function between successive iterations is commonly adopted to monitor whether convergence has been achieved. However, it is not efficient to use this criterion in the above $\mathrm{OC}$ algorithm because the objective function still oscillates slightly even when convergence is approached, taking a larger number of iterations to reach convergence. A more efficient strategy is to start recording the design variables and the objective function for the next 10 iterative steps once the relative changes in the design variables are all within 10 per cent. Out of the 10 recorded designs, the one with the smallest objective function is taken as the optimal design. Although the 10-per cent tolerance may seem large, this strategy does produce satisfactory results, as seen in the numerical examples below. Of course, if more accurate solutions are desired, the 10 per cent threshold can be reduced.

\subsection{Summary of the $O C$ algorithm}

The OC algorithm discussed above is summarized as follows:

1. Assume initial design $\mathbf{A}^{(0)}$. Let the initial accelerating coefficient $\beta^{(0)}=1.8$, reduction factor $\gamma=0.7$, and iteration counter $k=0$.

2. Solve the equilibrium equations: $\mathbf{K U}=\mathbf{P}_{0}$ (Equation (3); assume $\mathbf{P}=\mathbf{P}_{0}$ ). Note that $\mathbf{K}$ should be denoted by $\mathbf{K}_{0}$ and $\mathbf{U}$ by $\mathbf{U}_{0}$ for $k=0$.

3. Find the most active constraint and use it to define the scaling factor $s^{(k)}$ (Equation (41)).

4. Scale the design variables and displacements (Equations (42) and (43)).

5. Check the side constraints (Equations (44) and (45)).

6. Check convergence $(k>1)$ :

(a) If $\left|\left(\bar{A}_{i}^{(k)}-A_{i}^{(k-1)}\right) / \bar{A}_{i}^{(k)}\right| \geqslant 0.1$ for any $i$, then ICOUNT $=0$.

(b) If $\left|\left(\bar{A}_{i}^{(k)}-A_{i}^{(k-1)}\right) / \bar{A}_{i}^{(k)}\right|<0.1$ for all $i$, then ICOUNT $=$ ICOUNT +1 , start recording the values of the objective function and the design variables. If ICOUNT $=11$, go to Step 15. 
7. Evaluate the objective function $f\left(\overline{\mathbf{A}}^{(k)}\right)$.

8. If $k>2$, check the sign of $\left(f^{(k)}-f^{(k-1)}\right)\left(f^{(k-1)}-f^{(k-2)}\right)$. If it is negative, the values of the objective function are oscillatory. In this case, $\beta^{(k+1)}=\gamma \beta^{(k)}$; otherwise $\beta^{(k+1)}=\beta^{(k)}$. This will reduce the oscillation.

9. Compute the Lagrange multipliers associated with the inequality constraints. The only nonzero Lagrange multiplier is the one associated with the most active constraint (Section 4.3).

10. Calculate the Lagrange multipliers associated with the equality constraints (Equation (46)).

11. Calculate the residual $R_{i}^{(k)}$ and optimality index $\Omega_{i}^{(k)}$ (Equations (48)-(50)).

12. Update the areas (Equation (47)).

13. Check the side constraints as in Step 5.

14. Let $k=k+1$ and go to Step 2 .

15. Find the optimal solution from the last 10 recorded iterative steps. The optimal design is the one with the smallest objective function.

During each design cycle, there are two sets of equations, including those in Steps 2 and 10, that need to be solved. The conventional approach adopts the triple factoring method to solve them, whereas the proposed approach adopts the reduced basis method.

\section{NUMERICAL RESULTS AND DISCUSSIONS}

Five example problems are discussed in this section to verify the proposed reanalysis-based optimal design procedures using the MP and OC methods described before. The first three and the fifth examples, being of small scale, are mainly used to demonstrate that the accuracy of the optimal solutions obtained based on the proposed reanalysis technique is acceptable. The fourth example, a larger system, is used to discuss the efficiency. Also, the first four examples are concerned with sizing design, while the fifth example deals with configuration design of a truss. For this configuration design problem, only the MP method is adopted since the adopted OC method is applicable only for sizing design problem.

\subsection{Example 1: 10-bar truss}

The 10-bar truss shown in Figure 1 is a classical example in structural optimization, where $L=360$ in and $P=10^{5} \mathrm{lb}$. Other data are given as follows. Young's modulus $E=10^{7} \mathrm{psi}$; density $\rho=0.1 \mathrm{lb} / \mathrm{in}^{3}$; allowable tensile and compressive stresses for all members are both $25 \mathrm{ksi}$; the allowable displacements for all nodes are \pm 2 in along the $X$ and $Y$ directions; the lower bound and the initial value for all cross-sectional areas are 0.1 and $35 \mathrm{in}^{2}$, respectively.

The results are summarized in Table III. The conventional MP approach yields the same results as those reported in Reference [21]. The other approaches also provide very good results; the accuracy of the total mass is very good, although the accuracy of the sixth design variable is not good. For all the four methods, the optimal solutions have only two active constraints including the displacements at nodes 3 and 6 in the $Y$ direction and there are no constraints that are violated. These active constraints agree with those given in Reference [21]. The average number of basis vectors in Table III and later example problems is defined as

$$
\bar{m}=\frac{\sum_{i=1}^{N_{r}} m_{i}}{N_{r}}
$$




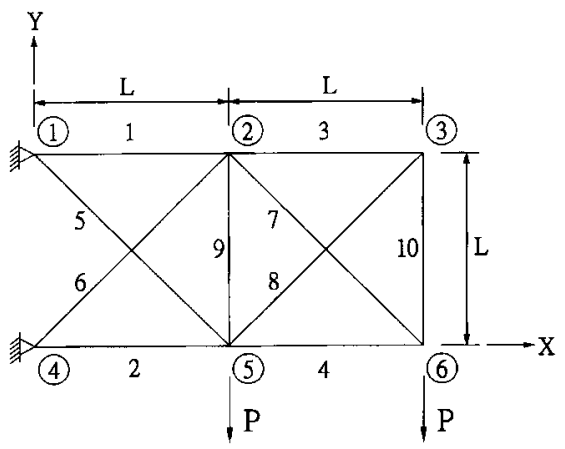

Figure 1. 10-bar truss.

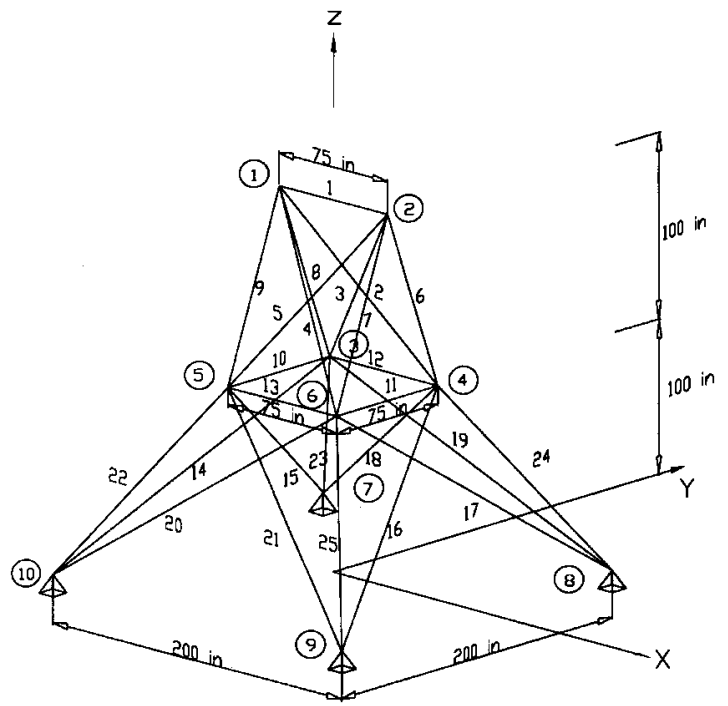

Figure 2. 25-bar truss.

Table III. Optimal designs for the 10-bar truss.

\begin{tabular}{lrrrrr}
\hline & \multicolumn{5}{c}{ Cross-sectional areas $\left(\right.$ in $\left.^{2}\right)$} \\
\cline { 2 - 6 } Member & \multicolumn{4}{c}{ MP } & OC \\
no. & 30.73 & 30.40 & 30.58 & 30.57 & 30.73 \\
& (Conven.) & (Present) & (Conven.) & (Present) & Reference [21] \\
2 & 23.94 & 22.93 & 23.07 & 23.15 & 23.94 \\
3 & 0.10 & 0.10 & 0.10 & 0.10 & 0.10 \\
4 & 14.37 & 15.37 & 15.21 & 15.13 & 14.73 \\
5 & 8.54 & 7.50 & 7.55 & 7.56 & 8.54 \\
6 & 20.95 & 21.20 & 21.26 & 21.08 & 20.95 \\
7 & 20.83 & 21.48 & 21.51 & 21.41 & 20.84 \\
8 & 0.10 & 0.10 & 0.10 & 0.10 & 0.10 \\
9 & 0.10 & 0.10 & 0.10 & 0.10 & 0.10 \\
10 & 0.10 & 0.48 & 0.43 & 0.72 & 0.10 \\
Mass (lb) & 5076.67 & 5057.23 & 5063.14 & 5065.01 & 5076.67 \\
Iteration statistics $\left(N_{1} / N_{2}\right)^{*}$ & $(53 / 45)$ & $(51 / 37)$ & $(21 / 23)$ & $(23 / 28)$ & - \\
Average basis no. $\bar{m}$ & - & 4.89 & - & 4.29 & - \\
\hline
\end{tabular}

* $\left(N_{1} / N_{2}\right)$. For MP method: $N_{1}$ is total number of calculating the objective function and constraints; $N_{2}$ the total number of calculating gradients of the objective function and constraints. For OC method: $N_{1}$ is the iteration step at which the objective function is minimum; $N_{2}$ the total number of iterations.

where the summation is taken over all the reanalyses carried out using the reduced basis method. As can be seen from Table III $\bar{m}$ is small, indicating that the adopted basis vectors are very accurate.

As discussed in Section 3.3, for the reanalysis-based MP (i.e. present MP) method, the sensitivities of nodal displacements are also obtained using the reanalysis method. From Table III, 
REANALYSIS-BASED OPTIMAL DESIGN OF TRUSSES

Table IV. Displacement sensitivities for the 10-bar truss.

\begin{tabular}{|c|c|c|c|c|c|c|c|c|c|}
\hline \multirow[b]{2}{*}{$\begin{array}{l}\text { Design var. } \\
\text { (Meth.) }\end{array}$} & \multicolumn{9}{|c|}{ Sensitivities of displacements* $\left(10^{-5} /\right.$ in $)$} \\
\hline & $U_{1}^{\prime}$ & $U_{2}^{\prime}$ & $U_{3}^{\prime}$ & $U_{4}^{\prime}$ & $U_{5}^{\prime}$ & $U_{6}^{\prime}$ & $U_{7}^{\prime}$ & $U_{8}^{\prime}$ & $\begin{array}{c}\text { Basis } \\
\text { no. }\end{array}$ \\
\hline$A_{1}$ (Conven.) & -68.52 & 45.63 & -68.74 & 118.7 & 8.092 & 40.53 & 7.316 & 120.2 & - \\
\hline$A_{1}$ (Present) & -68.52 & 45.58 & -68.74 & 118.6 & 8.110 & 40.52 & 7.404 & 120.2 & 4 \\
\hline$A_{2}$ (Conven.) & -25.01 & 315.2 & -22.17 & 1176 & 791.1 & 379.8 & 800.9 & 1156 & - \\
\hline$A_{2}$ (Present) & -25.02 & 315.6 & -22.27 & 1177 & 791.0 & 379.9 & 800.3 & 1157 & 5 \\
\hline$A_{3}$ (Conven.) & -0.030 & 0.375 & -8.776 & 5.946 & -0.122 & -0.612 & 1.114 & 3.451 & - \\
\hline$A_{3}$ (Present) & -0.030 & 0.367 & -8.776 & 5.943 & -0.119 & -0.616 & 1.122 & 3.449 & 5 \\
\hline$A_{4}$ (Conven.) & 0.788 & -9.926 & -8.657 & 83.49 & 3.231 & 16.19 & 211.2 & 149.5 & - \\
\hline$A_{4}$ (Present) & 0.641 & -9.681 & -8.791 & 83.27 & 3.196 & 16.54 & 210.7 & 149.0 & 5 \\
\hline$A_{5}$ (Conven.) & -59.46 & 749.2 & -52.70 & 671.9 & -243.9 & 902.8 & -220.5 & 624.7 & - \\
\hline$A_{5}$ (Present) & -59.40 & 750.0 & -51.47 & 670.8 & -244.2 & 902.9 & -219.4 & 626.9 & 4 \\
\hline$A_{6}$ (Conven.) & 52.72 & 1219 & 46.73 & 1288 & 216.2 & 1083 & 195.5 & 1330 & - \\
\hline$A_{6}$ (Present) & 52.74 & 1219 & 46.94 & 1288 & 217.0 & 1084 & 197.4 & 1330 & 5 \\
\hline$A_{7}$ (Conven.) & 0.680 & -8.565 & -7.469 & 72.04 & 2.788 & 13.96 & -25.42 & 129.0 & - \\
\hline$A_{7}$ (Present) & 0.680 & -8.515 & -7.472 & 72.10 & 2.771 & 14.03 & -25.43 & 129.0 & 6 \\
\hline$A_{8}$ (Conven.) & -0.363 & 4.570 & 3.986 & 72.38 & -1.488 & -7.452 & 13.56 & 42.01 & - \\
\hline$A_{8}$ (Present) & -0.352 & 4.398 & 3.994 & 72.32 & -1.431 & -7.495 & 13.74 & 41.99 & 5 \\
\hline$A_{9}$ (Conven.) & 3.872 & -48.79 & 9.520 & 52.76 & 15.88 & 79.55 & 35.43 & 13.31 & - \\
\hline$A_{9}$ (Present) & 3.877 & -48.77 & 9.538 & 52.91 & 15.87 & 79.80 & 35.47 & 13.42 & 6 \\
\hline$A_{10}$ (Conven.) & -1.453 & 18.31 & 15.97 & -154.0 & -5.961 & -29.86 & 54.35 & 168.3 & - \\
\hline$A_{10}$ (Present) & -1.744 & 19.44 & 15.61 & -152.8 & -6.257 & -28.89 & 53.67 & 168.6 & 4 \\
\hline
\end{tabular}

${ }^{*}$ Evaluated at $\mathbf{A}^{\mathrm{T}}=\langle 34.13,8.32,35.12,10.15,5.87,8.32,18.37,16.93,11.67,5.03\rangle \mathrm{in}^{2}$.

the present MP method delivers very good results, which indicates that the sensitivities of nodal displacements obtained in this way are also very accurate. In fact, not only for this example, but also for other examples presented in the following, the reanalysis-based sensitivities are very accurate. Take this 10-bar truss as an example. After 10 design cycles for the present MP method, the cross-sectional areas become

$$
\mathbf{A}^{\mathrm{T}}=\langle 34.13,8.32,35.12,10.15,5.87,8.32,18.37,16.93,11.67,5.03\rangle \quad\left(\text { unit: }^{2} \text { in }^{2}\right)
$$

The displacement sensitivities corresponding to this design, obtained using the conventional method (solving Equation (23) directly based on the triple factoring method) and the present (reanalysisbased) method are compared in Table IV. In this table, $U_{i}^{\prime}(i=1,2, \ldots, 8)$ represents the sensitivities of $U_{i}$ with respect to the cross-sectional areas, where $U_{1}, U_{3}, U_{5}$, and $U_{7}$ are, respectively, the displacements at nodes 2, 3, 5, and 6 in the $X$ direction, and $U_{2}, U_{4}, U_{6}$, and $U_{8}$ are the displacements in the $Y$ direction. It can be seen clearly from Table IV that the reanalysis-based displacement sensitivities are very accurate. Also shown in this table is the number of basis vectors required to satisfy the convergence criterion of Equation (11), where notice again that this study uses $e_{\mathrm{c}}=0.01$.

\subsection{Example 2: 25-bar truss}

The 25-bar truss shown in Figure 2 is also a commonly used benchmark problem. The structure is designed against two load cases that are listed in Table V. Material properties are: $E=10^{7}$ psi 
Table V. Nodal load components (lb) for the 25-bar truss.

\begin{tabular}{lcccc}
\hline Load case & Node & $X$ & $Y$ & $Z$ \\
\hline 1 & 1 & 1000 & 10000 & -5000 \\
& 2 & 0 & 10000 & -5000 \\
& 3 & 500 & 0 & 0 \\
& 6 & 500 & 0 & 0 \\
2 & 1 & 0 & 20000 & -5000 \\
& 2 & 0 & -20000 & -5000 \\
\hline
\end{tabular}

Table VI. Allowable compressive stresses and optimal designs for the 25-bar truss.

\begin{tabular}{lcccccccc}
\hline & & \multicolumn{7}{c}{ Cross-sectional areas $\left(\mathrm{in}^{2}\right)$} \\
\cline { 3 - 9 } $\begin{array}{l}\text { Design } \\
\text { variable }\end{array}$ & $\begin{array}{c}\text { Member } \\
\text { no. }\end{array}$ & $\begin{array}{c}\text { Allowable } \\
\text { comp. (psi) }\end{array}$ & $\begin{array}{c}\text { MP } \\
\text { (Conven.) }\end{array}$ & (Present) & $\begin{array}{c}\text { OC } \\
\text { (Conven.) }\end{array}$ & (Present) & $\begin{array}{c}\text { Reference } \\
{[21]}\end{array}$ & $\begin{array}{c}\text { Reference } \\
{[22]}\end{array}$ \\
\hline 1 & 1 & -35092 & 0.010 & 0.010 & 0.010 & 0.010 & 0.010 & 0.010 \\
2 & $2-5$ & -11590 & 1.932 & 1.933 & 1.924 & 1.924 & 1.987 & 1.987 \\
3 & $6-9$ & -17305 & 2.985 & 2.985 & 2.992 & 3.012 & 2.994 & 2.991 \\
4 & 10,11 & -35092 & 0.010 & 0.010 & 0.010 & 0.010 & 0.010 & 0.010 \\
5 & 12,13 & -35092 & 0.010 & 0.010 & 0.023 & 0.036 & 0.010 & 0.012 \\
6 & $14-17$ & -6759 & 0.684 & 0.684 & 0.685 & 0.693 & 0.684 & 0.683 \\
7 & $18-21$ & -6759 & 1.734 & 1.734 & 1.738 & 1.730 & 1.677 & 1.679 \\
8 & $22-25$ & -11082 & 2.651 & 2.651 & 2.648 & 2.687 & 2.662 & 2.664 \\
Mass $(1 \mathrm{lb})$ & - & - & 545.50 & 545.56 & 545.86 & 548.86 & 545.16 & 545.22 \\
$\left(N_{1} / N_{2}\right)$ & - & - & $(59 / 38)$ & $(58 / 36)$ & $(26 / 29)$ & $(22 / 23)$ & - & - \\
$\bar{m}$ & - & - & - & 4.55 & - & 6.42 & - & - \\
\hline
\end{tabular}

and $\rho=0.1 \mathrm{lb} / \mathrm{in}^{3}$. The lower bound and the initial value for each cross-sectional area are 0.01 and $10 \mathrm{in}^{2}$, respectively. The allowable displacements for nodes 1 and 2 are \pm 0.35 in along the $X$-, $Y$-, and $Z$-axis, whereas the allowable tensile stress is $40000 \mathrm{psi}$ for all members and the allowable compressive stress is listed in Table VI.

Optimal designs are compared in Table VI. Clearly, both the conventional and the reanalysisbased (present) MP methods yield excellent solutions, which are also in good agreement with those given in References [21, 22]. The conventional and present OC methods also provide very good optimal solutions in terms of the total mass, although the accuracy of the fifth design variable is not good. The optimal solutions obtained by the above four methods have the same active constraints as follows: the displacements at nodes 3 and 6 in the $Y$ direction for both load cases and the compressive stresses in members 19 and 20 for the second load case. The above active constraints are the same as those reported in Reference [21]. Also, there are no constraint violations. The average number of basis vectors, $\bar{m}$, is small for all cases.

\subsection{Example 3: 72-bar truss}

The 72-bar truss of Figure 3, where $b=60$ in, is designed against two load cases that are given in Table VII. Material properties are: $E=10^{7} \mathrm{psi}$ and density $\rho=0.1 \mathrm{lb} / \mathrm{in}^{3}$. The lower bound 


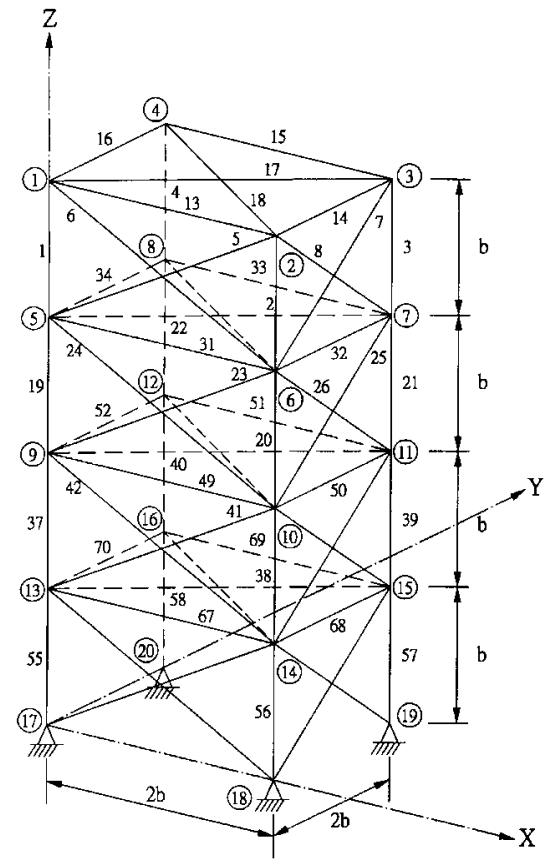

Figure 3. 72-bar truss.

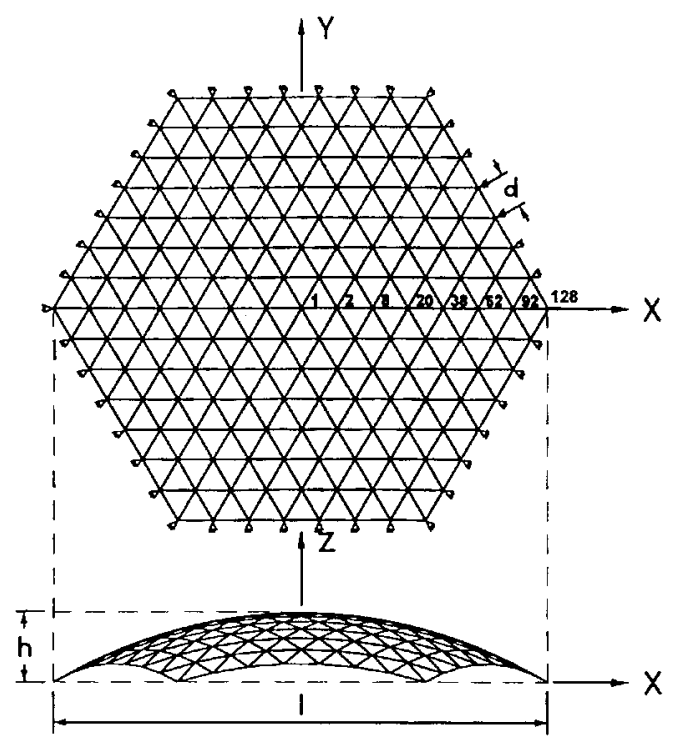

Figure 4. 462-bar truss.

Table VII. Nodal load components (lb) for the 72-bar truss.

\begin{tabular}{lcccc}
\hline Load case & Node & $X$ & $Y$ & $Z$ \\
\hline 1 & 1 & 5000 & 5000 & -5000 \\
2 & 1 & 0 & 0 & -5000 \\
& 2 & 0 & 0 & -5000 \\
& 3 & 0 & 0 & -5000 \\
& 4 & 0 & 0 & -5000 \\
\hline
\end{tabular}

and the initial value for each cross-sectional area are 0.1 and $10 \mathrm{in}^{2}$, respectively. The allowable displacements for nodes $1-4$ are \pm 0.25 in along the $X$-, $Y$-, and $Z$-axis, while the allowable tensile and compressive stresses are both $25 \mathrm{ksi}$ for all members.

Optimal designs are compared in Table VIII. Again, both the conventional and the present MP methods yield excellent solutions in comparison with those given in by Reference [21]. Also, the conventional and the present OC methods deliver very good solutions in terms of the total mass, even though the accuracy of the eighth design variable is not good. The final designs obtained by the above four methods have the following constraints active: the displacements at node 1 in both the $X$ and $Y$ directions for load case one, and the compressive stresses in members 1-4 for load case two. The above active constraints agree with those reported in Reference [21]. In addition, there are no constraints that are violated. Finally, $\bar{m}$ is again small. 
Table VIII. Optimal designs for the 72-bar truss.

\begin{tabular}{lcrrrrr}
\hline & & \multicolumn{5}{c}{ Cross-sectional areas (in $\left.{ }^{2}\right)$} \\
\cline { 3 - 7 } $\begin{array}{l}\text { Design } \\
\text { variable }\end{array}$ & $\begin{array}{c}\text { Member } \\
\text { no. }\end{array}$ & (Conven.) & MP Present) & (Conven.) & OC Present) & Reference [21] \\
\hline 1 & $1-4$ & 0.1565 & 0.1556 & 0.1562 & 0.1556 & 0.1571 \\
2 & $5-12$ & 0.5456 & 0.5446 & 0.5513 & 0.5530 & 0.5356 \\
3 & $13-16$ & 0.4104 & 0.4184 & 0.4051 & 0.4040 & 0.4096 \\
4 & 17,18 & 0.5697 & 0.5659 & 0.5462 & 0.5459 & 0.5693 \\
5 & $19-22$ & 0.5237 & 0.5409 & 0.5171 & 0.5166 & 0.5067 \\
6 & $23-30$ & 0.5171 & 0.5202 & 0.5150 & 0.5148 & 0.5200 \\
7 & $31-34$ & 0.1000 & 0.1000 & 0.1000 & 0.1006 & 0.1000 \\
8 & 35,36 & 0.1000 & 0.1000 & 0.1426 & 0.1432 & 0.1000 \\
9 & $37-40$ & 1.2684 & 1.2090 & 1.2731 & 1.2759 & 1.2801 \\
10 & $41-48$ & 0.5117 & 0.5162 & 0.5101 & 0.5095 & 0.5148 \\
11 & $49-52$ & 0.1000 & 0.1000 & 0.1000 & 0.1000 & 0.1000 \\
12 & 53,54 & 0.1000 & 0.1000 & 0.1000 & 0.1000 & 0.1000 \\
13 & $55-58$ & 1.8862 & 1.8154 & 1.8986 & 1.8983 & 1.8973 \\
14 & $59-66$ & 0.5123 & 0.5150 & 0.5114 & 0.5107 & 0.5158 \\
15 & $67-70$ & 0.1000 & 0.1000 & 0.1000 & 0.1000 & 0.1000 \\
16 & 71,72 & 0.1000 & 0.1000 & 0.1000 & 0.1000 & 0.1000 \\
Mass (1b) & - & 376.62 & 379.25 & 380.38 & 380.41 & 379.66 \\
$\left(N_{1} / N_{2}\right)$ & - & $(64 / 45)$ & $(40 / 25)$ & $(25 / 28)$ & $(25 / 28)$ & - \\
$\bar{m}$ & - & - & 5.07 & - & 6.24 & - \\
\hline
\end{tabular}

\subsection{Example 4: 462-bar truss}

Consider the space truss shown in Figure 4, where $h=1.489 \mathrm{~m}, l=10.5 \mathrm{~m}$, and $d=0.75 \mathrm{~m}$. The co-ordinates of the nodes can be generated according to $X^{2}+Y^{2}+(Z+8.511)^{2}=100$. This truss has 169 nodes, 462 members, and 381 degrees of freedom. The half-bandwidth of the stiffness matrix is 198 if the nodes are numbered from the inner to the outer hexagons as depicted in Figure 4; note that the boundary nodes are fixed in all directions. The material properties are $E=200 \mathrm{GPa}$, and $\rho=7650 \mathrm{~kg} / \mathrm{m}^{3}$.

Three design problems with different numbers of design variables are considered below. The $X-Y$ plane projection of the structure is composed of seven hexagons as depicted in Figure 4; we refer to the innermost one as the first and the outmost one as the seventh. Case 1 has two design variables, where the first design variable is the area of the members that belong to any of the seven hexagons and the second design variable is the area of the other members (i.e. braces). Case 2 has seven design variables; the first design variable is the area of the six members of the first hexagon and the six braces inside it and the second design variable is the area of the 12 members of the second hexagon and the 18 braces inside it but outside the first hexagon. The other design variables are defined sequentially following this rule. Case 3 has 14 design variables, which are defined in a way similar to case two, except that the braces have areas different from the areas of the members of the hexagons. Therefore, the seven odd design variables are the areas of the seven hexagons and the seven even design variables are the areas of the braces; both even and odd design variables are numbered sequentially from the inner to the outer hexagons.

For the above three cases having different numbers of design variables, the structure is designed to resist $100 \mathrm{~N}$ loads applied at all nodes. The lower and upper bounds for all cross-sectional areas 
Table IX. Optimal designs for the 462-bar truss with two design variables.

\begin{tabular}{|c|c|c|c|c|}
\hline \multirow{3}{*}{$\begin{array}{l}\text { Design } \\
\text { variable }\end{array}$} & \multicolumn{4}{|c|}{ Cross-sectional areas $\left(\mathrm{in}^{2}\right)$} \\
\hline & \multicolumn{2}{|c|}{ MP } & \multicolumn{2}{|c|}{$\mathrm{OC}$} \\
\hline & (Conven.) & (Present) & (Conven.) & (Present) \\
\hline 1 & 23.267 & 23.267 & 25.000 & 25.000 \\
\hline 2 & 22.002 & 22.002 & 21.998 & 21.994 \\
\hline Mass (kg) & 62.42 & 62.42 & 65.48 & 65.47 \\
\hline$\left(N_{1} / N_{2}\right)$ & $(20 / 10)$ & $(19 / 10)$ & $(2 / 12)$ & $(2 / 12)$ \\
\hline CPU time (s) & 131 & 70 & 135 & 38 \\
\hline $\bar{m}$ & - & 2.25 & - & 2.70 \\
\hline
\end{tabular}

Table X. Optimal designs for the 462-bar truss with seven design variables.

\begin{tabular}{lcccc}
\hline & \multicolumn{4}{c}{ Cross-sectional areas $\left(\mathrm{in}^{2}\right)$} \\
\cline { 2 - 5 } $\begin{array}{l}\text { Design } \\
\text { variable }\end{array}$ & (Conven.) & MP & OC & \\
\hline 1 & 22.206 & 22.206 & 23.063 & 23.063 \\
2 & 22.330 & 22.330 & 22.987 & 22.985 \\
3 & 22.563 & 22.563 & 22.564 & 22.562 \\
4 & 22.874 & 22.874 & 25.000 & 25.000 \\
5 & 23.194 & 23.194 & 23.380 & 23.378 \\
6 & 23.344 & 23.344 & 23.754 & 23.753 \\
7 & 23.372 & 23.372 & 23.841 & 23.840 \\
Mass $(\mathrm{kg})$ & 63.16 & 63.16 & 64.84 & 64.83 \\
$\left(N_{1} / N_{2}\right)$ & $(15 / 9)$ & $(13 / 8)$ & $(5 / 12)$ & $(5 / 12)$ \\
CPU time (s) & 121 & 82 & 147 & 48 \\
$\bar{m}$ & - & 1.34 & - & 2.57 \\
\hline
\end{tabular}

are 5 and $25 \mathrm{~mm}^{2}$, respectively. The allowable stress is $20 \mathrm{MPa}$ for both tension and compression. The optimal designs obtained using the conventional and proposed methods are compared in Tables IX-XI, where the CPU times are counted on an HP 9000/715 workstation. It can be seen from these tables that both the reanalysis-based MP and OC methods are more efficient than the conventional ones. However, the efficiency of the reanalysis-based MP method becomes insignificant as the number of design variables increases, as explained in the last paragraph of Section 3. The efficiency for the reanalysis-based OC method is higher than the reanalysis-based MP method and also it is only slightly affected as the number of design variables increases.

\subsection{Example 5: configuration design}

The last example is concerned with configuration design for the 18-bar planar truss of Figure 5 . The member areas are linked as follows: $A_{1}=A_{4}=A_{8}=A_{12}=A_{16} ; A_{2}=A_{6}=A_{10}=A_{14}=A_{18}$; $A_{3}=A_{7}=A_{11}=A_{15} ; A_{5}=A_{9}=A_{13}=A_{17}$. The configuration variables are the following co-ordinates: $X_{3}, Y_{3}, X_{5}, Y_{5}, X_{7}, Y_{7}, X_{9}$, and $Y_{9}$. Totally, there are four area variables and eight coordinate variables. 
Table XI. Optimal designs for the 462-bar truss with fourteen design variables.

\begin{tabular}{lcccc}
\hline & \multicolumn{4}{c}{ Cross-sectional areas $\left(\mathrm{in}^{2}\right)$} \\
\cline { 2 - 5 } $\begin{array}{l}\text { Design } \\
\text { variable }\end{array}$ & MP & OC \\
\hline 1 & 22.206 & 22.174 & 22.317 & 22.312 \\
2 & 22.002 & 21.965 & 22.281 & 22.276 \\
3 & 22.328 & 22.300 & 22.340 & 22.335 \\
4 & 21.440 & 21.440 & 22.102 & 22.097 \\
5 & 22.547 & 22.529 & 25.000 & 25.000 \\
6 & 20.513 & 20.490 & 21.531 & 21.526 \\
7 & 22.806 & 22.800 & 22.804 & 22.799 \\
8 & 19.217 & 19.233 & 20.171 & 20.167 \\
9 & 22.985 & 22.992 & 23.464 & 23.459 \\
10 & 17.559 & 17.603 & 17.569 & 17.565 \\
11 & 22.965 & 22.970 & 24.417 & 24.412 \\
12 & 15.566 & 15.530 & 17.137 & 17.135 \\
13 & 23.015 & 23.024 & 24.937 & 24.937 \\
14 & 5.000 & 5.000 & 5.00 & 5.00 \\
Mass $(\mathrm{kg})$ & 56.30 & 56.30 & 59.09 & 59.08 \\
$\left(N_{1} / N_{2}\right)$ & $(30 / 13)$ & $(28 / 12)$ & $(3 / 13)$ & $(3 / 13)$ \\
CPU time (s) & 290 & 248 & 181 & 67 \\
$\bar{m}$ & - & 1.72 & - & 3.04 \\
\hline
\end{tabular}

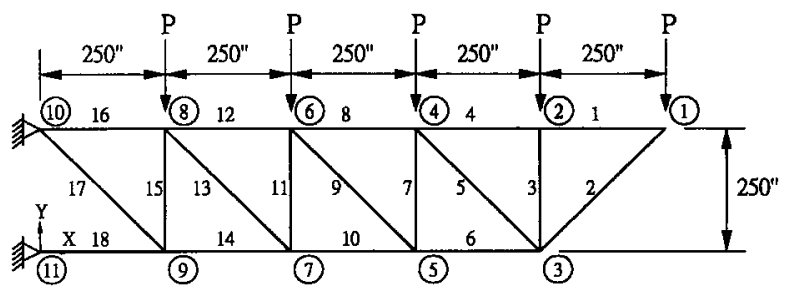

Figure 5. 18-bar truss.

The applied loads are shown in Figure 5, where $P=20$ kips. The material properties are: $E=10^{7}$ psi and $\rho=0.1 \mathrm{lb} / \mathrm{in}^{3}$. The lower bound for all cross-sectional areas is $0.1 \mathrm{in}^{2}$. The allowable stress is $20 \mathrm{ksi}$ for both tension and compression. The data for the initial and final designs are given in Table XII. Clearly, the total masses obtained using the conventional and the reanalysis-based MP methods are in good agreement with that given in Reference [23], although the values of the design variables are slightly different. The optimal solutions obtained by the above two methods have nine active constraints including the compressive stresses in members $3,7,11,15$, and 18 and the tensile stresses in members $4,12,16$, and 17 . There are no constraint violations. The active constraints corresponding to the optimal solution given in Reference [23] are the same as those of this study except that the tensile stress constraint for member 4 is inactive, which is only $12.78 \mathrm{ksi}$. The average number of basis vectors is again very small. 
Table XII. Initial design and optimal designs for the 18-bar truss.

\begin{tabular}{lrrrr}
\hline $\begin{array}{l}\text { Design } \\
\text { variable }\end{array}$ & $\begin{array}{l}\text { Initial } \\
\text { design }\end{array}$ & $\begin{array}{c}\text { MP } \\
\text { (Conven.) }\end{array}$ & (Present) & Reference [23] \\
\hline$A_{1}\left(\right.$ in $\left.^{2}\right)$ & 10.0 & 11.41 & 11.44 & 10.71 \\
$A_{2}\left(\right.$ in $\left.^{2}\right)$ & 15.0 & 15.21 & 15.21 & 15.19 \\
$A_{3}\left(\right.$ in $\left.^{2}\right)$ & 1.0 & 1.33 & 1.25 & 1.94 \\
$A_{5}\left(\right.$ in $\left.^{2}\right)$ & 7.07 & 4.35 & 4.33 & 5.19 \\
$X_{3}$ (in) & 1000.0 & 977.4 & 981.4 & 881.4 \\
$Y_{3}$ (in) & 0.0 & 224.1 & 225.1 & 178.8 \\
$X_{5}$ (in) & 750.0 & 595.1 & 632.6 & 628.9 \\
$Y_{5}$ (in) & 0.0 & 135.6 & 147.4 & 124.9 \\
$X_{7}$ (in) & 500.0 & 402.2 & 421.4 & 390.5 \\
$Y_{7}$ (in) & 0.0 & 84.3 & 91.4 & 66.8 \\
$X_{9}$ (in) & 250.0 & 292.3 & 289.9 & 313.2 \\
$Y_{9}$ (in) & 0.0 & 49.5 & 48.9 & 45.0 \\
Mass (lb) & - & 3871.3 & 3869.6 & 3906.8 \\
$\left(N_{1} / N_{2}\right)$ & - & $(104 / 93)$ & $(70 / 89)$ & - \\
$\bar{m}$ & - & - & 4.78 & - \\
\hline
\end{tabular}

\section{CONCLUSIONS}

This paper has presented a reduced basis method for structural reanalysis. Some features of the method make it suitable for use in design optimization. Especially, a computation-inexpensive error measure, introduced to adaptively determine whether the solution converges, is made possible through the use of the Gram-Schmidt orthonormalization procedure. Procedures for implementing the reanalysis technique with both the MP and OC methods have also been detailed. According to the presented example problems on optimal design of trusses and the particular MP and OC optimizers chosen in this study, the following points can be addressed. First, the accuracy of the optimal designs obtained on the basis of the reanalysis technique is affected only very slightly, which is acceptable from the engineering point of view. Second, for problems of large scale, the reanalysis method does speed up the design process. Third, the speed increase of the design process for the reanalysis-based MP method will decrease as the number of design variables increases, while it will not decrease for the reanalysis-based OC method.

\section{APPENDIX}

The stiffness of a spatial truss element is of the form

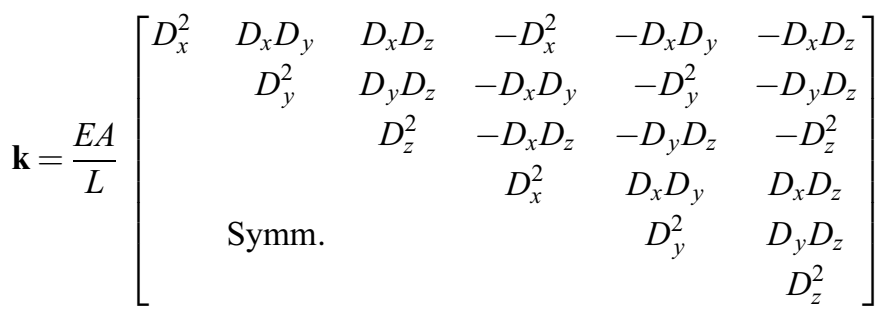


In the above, $E$ is Young's modulus, $A$ the cross-sectional area, $L$ the element's length, $D_{x}=$ $X_{2}-X_{1}, D_{y}=Y_{2}-Y_{1}$, and $D_{z}=Z_{2}-Z_{1}$, where $\left(X_{1}, Y_{1}, Z_{1}\right)$ and $\left(X_{2}, Y_{2}, Z_{2}\right)$ denote the coordinates of the end nodes of the element.

\section{ACKNOWLEDGEMENTS}

This research was sponsored by the National Science Council of the Republic of China under Grant Numbers NSC-85-2211-E-002-013 and NSC-86-2221-E-002-076.

\section{REFERENCES}

1. Kirsch U. Structural Optimization: Fundamentals and Applications. Springer: New York, 1993.

2. Leu L-J, Mukherjee S. Sensitivity analysis and shape optimization in nonlinear solid mechanics. Engineering Analysis with Boundary Elements 1993; 12:251-260.

3. Leu L-J. Sensitivity analysis and optimization in nonlinear solid mechanics. Ph.D. Dissertation, Cornell University, Ithaca, New York, 1994.

4. Taye S. Norm optimization techniques for improved structural reanalysis. Computers and Stuctures 1991; 41: 1341-1347.

5. Kirsch U. Reduced basis approximations of structural displacements for optimal design. AIAA Journal 1991; 29: $1751-1758$.

6. Kirsch U. Improved stiffness-based first-order approximations for structural optimization. AIAA Journal 1995; 33: $143-150$.

7. Kane JH, Kumar BLK, Gallagher GH. Boundary-element iterative reanalysis for continuum structures. Journal of Engineering Mechanics ASCE 1990; 116:2293-2309.

8. Saigal S. Iteration schemes for improved convergence in boundary element reanalysis. Computer Methods in Applied Mechanics and Engineering 1990; 84:97-107.

9. Leu L-J. A reduction method for boundary element reanalysis. Computer Methods in Applied Mechanics and Engineering 1999; 178:125-139.

10. Leu L-J. Shape optimization by the boundary element method with a reduced basis reanalysis technique. Structural Engineering and Mechanics 1999; 8:73-84.

11. Tong P, Rossettos JN. Finite-Element Method: Basic Technique and Implementation. MIT Press: Cambridge, MA, 1977.

12. Greenberg MD. Foundations of Applied Mathematics. Prentice-Hall: Englewood Cliffs, NJ, 1978.

13. Huang C-W. Applications of reduction methods in structural optimization and nonlinear analysis. M.Sc. Thesis, Department of Civil Engineering, National Taiwan University, Taipei, Taiwan, ROC, 1996 (in Chinese).

14. Kirsch U. Efficient reanalysis for topological optimization. Structural Optimization 1993; 6:143-150.

15. IMSL. MATH/LIBRARY, Fortran Subroutines for Mathematical Applications, vol. 2, Visual Numerics, 1994; 925-932.

16. Schittowski K. NLPQL: a FORTRAN subroutine solving constrained nonlinear programming problems. Annals of Operations Research 1985; 5:485-500.

17. Gutkowski W, Bauer J, Iwanow Z. Discrete structural optimization. Computer Methods in Applied Mechanics and Engineering 1985; 51:71-78.

18. Gutkowski W, Bauer J, Iwanow Z. Explicit formulation of Kuhn-Tucker necessary conditions in structural optimization. Computers and Structures 1990; 37:753-758.

19. Vanderplaats GN. Numerical Optimization Techniques for Engineering Design with Applications. McGraw-Hill: New York, 1984

20. Khan MR. Optimality criterion techniques applied to frames having general cross-sectional relationships. AIAA Journal 1984; 22:669-676.

21. Zhou M, Rozvany GIN. DCOC: an optimality criteria method for large systems, Part II: algorithm. Structural and Optimization 1993; 6:250-262.

22. Haftka RT, Gürdal Z. Elements of Structural Optimization, (3rd edn). Kluwer Academic Publishers: Dordrecht, 1992.

23. Hansen SR, Vanderplaats GN. Approximation method for configuration optimization of trusses. AIAA Journal 1990; 28:161-168. 